\title{
Manganese availability regulates Mn(III)-driven organic matter degradation at oxic-anoxic interfaces
}

\author{
NATHAN A CHIN ${ }^{1}$, XIAO-JUN ALLEN LIU ${ }^{1}$, LUIZ \\ DOMEIGNOZ HORTA ${ }^{1,2}$, KRISTEN DEANGELIS ${ }^{1}$ AND \\ MARCO KEILUWEIT ${ }^{1}$ \\ ${ }^{1}$ University of Massachusetts - Amherst \\ ${ }^{2}$ University of Zurich \\ Presenting Author: nathanchin@umass.edu
}

Reactive manganese $(\mathrm{Mn})$ phases have recently emerged as a critical driver of organic matter degradation in soil and sediment environments, affecting both $\mathrm{CO}_{2}$ emissions and carbon storage. Recent evidence suggests that oxic-anoxic interfaces act as critical hotspots for the formation of reactive $\mathrm{Mn}(\mathrm{III})$ species and associated organic matter degradation. Yet, the extent to which microbially-mediated, Mn(III)-driven organic matter oxidation depends on $\mathrm{Mn}$ availability remains largely unknown. In this study, we quantified how variations in Mn bioavailability affect rates and pathways of $\mathrm{Mn}(\mathrm{III})$-driven organic matter degradation along oxic-anoxic interfaces. To this end, we established soil redox gradients in diffusion reactors and varied the $\mathrm{Mn}$ (II) supply toward the oxic-anoxic interface. Over a 12-week incubation, we quantified variations in $\mathrm{Mn}$ (II) oxidation rates, microbial abundance and gene expression, and organic matter degradation across the redox gradient. Pore water analyses, Mn XANES, and wet-chemical extractions indicated that Mn(III) formation at the oxic-anoxic interface peaked after 4 weeks of incubation and increased with increasing $\mathrm{Mn}$ availability. Quantitative PCR showed greater fungal:bacterial ratios with increasing $\mathrm{Mn}$ availability, while metatranscriptomics illuminated presumed Mn(II)-oxidizing enzyme dynamics. Carbon XANES, microbial respiration rates, and phenol oxidation potential assays showed that organic matter degradation increased with $\mathrm{Mn}$ availability, with peaks coinciding with $\mathrm{Mn}$ (III) formation at the oxic-anoxic interfaces. Combined, our results showed how Mn availability regulates microbial $\mathrm{Mn}$ (II) oxidation rates along oxic-anoxic interfaces and thus the potential for Mn(III)-driven organic matter degradation. Our findings suggest that ecosystem-scale Mn bioavailability controls the extent to which oxic-anoxic interfaces act as hotspots for $\mathrm{Mn}(\mathrm{III})$-mediated organic matter degradation. 